International Journal of Pure and Applied Mathematics

Volume 82 No. 4 2013, 573-580

ISSN: 1311-8080 (printed version); ISSN: 1314-3395 (on-line version)

url: http://www.ijpam.eu

doi: http://dx.doi.org/10.12732/ijpam.v82i4.6

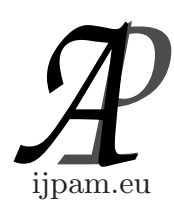

\title{
NORM INEQUALITIES FOR SEQUENCES OF BOUNDED LINEAR OPERATORS IN HILBERT SPACES
}

\author{
Mohammed Al-Dolat ${ }^{1}$, Mohammed $\mathrm{Ali}^{2} \S$ \\ ${ }^{1,2}$ Department of Mathematics and Statistics \\ Jordan University of Science and Technology \\ Irbid, JORDAN
}

Abstract: Let $B(H)$ be the space of all bounded linear operators on a complex Hilbert space $H$, and let $A_{1}, \ldots, A_{n} \in B(H)$. In this article, we obtain new upper bounds for $\left\|\sum_{j=1}^{n} A_{j}\right\|$. Moreover, we establish and generalize inequalities for the operator norm of sums of bounded linear operators in Hilbert spaces.

AMS Subject Classification: 47A05, 47A12

Key Words: operator norm, Hilbert space

\section{Introduction}

Let $H$ be a complex Hilbert space with inner product $\langle.,$.$\rangle , and let B(H)$ be the space of all bounded linear operators on $H$. For $A \in B(H)$, let $A^{*}$ denote the adjoint operator of $A$, and $\|A\|$ denote the usual operator norm given by

$$
\|A\|=\sup _{\|x\|=1}\|A x\|
$$

where $\|A x\|=\langle A x, A x\rangle^{\frac{1}{2}}$.

For $A_{1}, \ldots, A_{n} \in B(H)$, several inequalities that provide alternative upper bounds for $\left\|\sum_{j=1}^{n} A_{j}\right\|$ have been established by many authors (we refer the read-

Received: September 9, 2012

(C) 2013 Academic Publications, Ltd.

$\S_{\text {Correspondence author }}$ url: www.acadpubl.eu 
ers to [1], [2], [3], as well as [5], and the references therein). For example, [3] established various inequalities which give upper bounds for $\left\|\sum_{j=1}^{n} A_{j}\right\|$. Subsequently, [2] used a worthy technique to obtain new upper bounds for $\left\|\sum_{j=1}^{n} A_{j}\right\|$. Recently, [1] applied different approaches to improve these upper bounds. Moreover, [1] got two refinements of the generalized triangle inequality for $\left\|\sum_{j=1}^{n} A_{j}\right\|$. Specifically, he proved the following inequalities:

$$
\begin{gathered}
\left\|\sum_{k=1}^{n} A_{k}\right\|^{2} \leq\left\|\sum_{k=1}^{n} A_{k}^{*} A_{k}\right\|+\frac{1}{2}\left\|\sum_{j=1}^{n} A_{j}^{*} \sum_{k=1}^{n} A_{k}-\sum_{k=1}^{n} A_{k}^{*} A_{k}\right\|^{2}+\frac{1}{2}, \\
\left\|\sum_{k=1}^{n} A_{k}\right\|^{2} \leq\left\|\sum_{k=1}^{n} A_{k}^{*} A_{k}\right\|+\frac{1}{2}\left\|(n-2) \sum_{k=1}^{n} A_{k}^{*} A_{k}+\sum_{k=1}^{n} A_{k}^{*} \sum_{k=1}^{n} A_{k}\right\|,
\end{gathered}
$$

and

$$
\left\|\sum_{k=1}^{n} A_{k}\right\|^{2} \leq\left\|\sum_{k=1}^{n} A_{k}^{*} A_{k}\right\|+\frac{1}{4}\left\|\sum_{1 \leq j \neq k \leq n} A_{j}^{*} A_{k}+I\right\|^{2} .
$$

The purpose of this work is to establish some new inequalities for the operator norm of sums of bounded linear operators in Hilbert spaces. Also, we generalize the inequalities (1) and (2). Moreover, we use a tranquil technique to attain a refinement of [Inequality (5), [7]].

\section{The Main Results}

In this section, we introduce and prove some new inequalities for the operator norm of sums of bounded linear operators in Hilbert spaces. Also, we improve and generalize some known results concerning $\left\|\sum_{j=1}^{n} A_{j}\right\| \leq \sum_{j=1}^{n}\left\|A_{j}\right\|$. The proofs of our sequels mainly depend on [1] as well as the following lemmas.

Lemma 1 ([4]). For $a, b \geq 0,0 \leq \alpha \leq 1$, and $r \neq 0$. Let $M_{r}(a, b, \alpha)=$ $\left(\alpha a^{r}+(1-\alpha) b^{r}\right)^{\frac{1}{r}}$ and $M_{0}(a, b, \alpha)=a^{\alpha} b^{1-\alpha}$. Then

$$
M_{r}(a, b, \alpha) \leq M_{s}(a, b, \alpha) \quad \text { for } r \leq s .
$$


Lemma 2 ([6]). Let $A \in B(H)$ be a positive operator, and let $x \in H$ be a unit vector. Then

$$
\langle A x, x\rangle^{r} \leq\left\langle A^{r} x, x\right\rangle \quad \text { for } r \geq 1 .
$$

Let us use this lemma to generalize inequality (1).

Lemma 3. Let $A_{1}, A_{2}, \ldots, A_{n} \in B(H)$ and $r \geq 1$. Then

$$
\left\|\sum_{k=1}^{n} A_{k}\right\|^{2} \leq\left\|\sum_{k=1}^{n} A_{k}^{*} A_{k}\right\|+\left(\frac{1}{2}+\frac{1}{2}\left\|\sum_{1 \leq j \neq k \leq n} A_{j}^{*} A_{k}\right\|^{2 r \quad \frac{1}{r}}\right)^{.}
$$

Proof. For any $x \in H$, Lemma 1 yields that

$$
\begin{aligned}
\left\|\sum_{k=1}^{n} A_{k} x\right\|^{2} & =\sum_{k=1}^{n}\left\langle A_{k}^{*} x, A_{k} x\right\rangle+\sum_{1 \leq j \neq k \leq n} \operatorname{Re}\left\langle A_{k} x, A_{j} x\right\rangle \\
& =\sum_{k=1}^{n}\left\langle A_{k}^{*} x, A_{k} x\right\rangle+\operatorname{Re}\left\langle\sum_{1 \leq j \neq k \leq n} A_{j}^{*} A_{k} x, x\right\rangle \\
& \leq \sum_{k=1}^{n}\left\langle A_{k}^{*} A_{k} x, x\right\rangle+\frac{1}{2}\left\|\sum_{1 \leq j \neq k \leq n} A_{j}^{*} A_{k} x\right\|^{2}+\frac{\|x\|^{2}}{2} \\
& \leq\left\langle\sum_{k=1}^{n} A_{k}^{*} A_{k} x, x\right\rangle+\left(\frac{1}{2}\left\|\sum_{1 \leq j \neq k \leq n} A_{j}^{*} A_{k} x\right\|^{2 r}+\frac{1}{2}\|x\|^{2 r} \|^{\frac{1}{r}} .\right.
\end{aligned}
$$

We complete the proof by taking the superemum over all unit vectors.

In the above lemma, take $r=1$, we get inequality (1).

As a direct consequence of Lemma 3 we obtain the following corollary.

Corollary 4. Let $A \in B(H)$ and $r \geq 1$. Then

$$
\|A\|^{2} \leq\left\|\frac{A^{*} A+A A^{*}}{2}\right\|+\left(\frac{1}{2}+\frac{1}{4}\left\|A^{*} A-A A^{*}\right\|^{2 r}\right)^{\frac{1}{r}} .
$$

Proof. For any bounded linear operator $A \in B(H)$, we let $A=B+i C$ be the cartesian decomposition of $A$. Since $B^{*}=B$ and $C^{*}=C$, we obtain that

$$
\|A\|^{2} \leq\left\|B^{*} B+(i C)^{*}(i C)\right\|+\left(\frac{1}{2}+\frac{1}{2}\left\|B^{*}(i C)+(i C)^{*} B\right\|^{2 r}\right)^{\frac{1}{r}}
$$




$$
\begin{aligned}
& =\left\|B^{2}+C^{2}\right\|+\left(\frac{1}{2}+\frac{1}{2}\|B C-C B\|^{2 r}\right)^{\frac{1}{r}} \\
& =\left\|\frac{A^{*} A+A A^{*}}{2}\right\|+\left(\frac{1}{2}+\frac{1}{4}\left\|A^{*} A-A A^{*}\right\|^{2 r}\right)^{\frac{1}{r}} .
\end{aligned}
$$

A straightforward technique plus Lemma 1 and Lemma 2, we reach the following theorem.

Theorem 5. Let $A_{1}, \ldots, A_{n} \in B(H), 0<\alpha<1$. Then for any $r \geq 1$,

$$
\begin{aligned}
\left\|\sum_{k=1}^{n} A_{k}\right\|^{2} & \leq\left\|\sum_{k=1}^{n} A_{k}^{*} A_{k}\right\| \\
& +\left\|\sum_{1 \leq j \neq k \leq n}\left[\alpha\left(\frac{1}{\sqrt[\alpha]{4}}\right)^{r} I+(1-\alpha)\left(\left(A_{k}^{*}+A_{j}^{*}\right)\left(A_{k}+A_{j}\right)\right)^{\frac{r}{1-\alpha}}\right]\right\|^{\frac{1}{r}} .
\end{aligned}
$$

Proof. Let $x \in H$ be any vector. By Lemmas 1-2, we acquire that

$$
\begin{aligned}
& \left\|\sum_{k=1}^{n} A_{k} x\right\|^{2}=\sum_{k=1}^{n}\left\langle A_{k}^{*} A_{k} x, x\right\rangle+\frac{1}{4} \sum_{1 \leq j \neq k \leq n}\left(\left\|\left(A_{k}+A_{j}\right) x\right\|^{2}-\left\|\left(A_{k}-A_{j}\right) x\right\|^{2}\right) \\
& \leq \sum_{k=1}^{n}\left\langle A_{k}^{*} A_{k} x, x\right\rangle+\frac{1}{4} \sum_{1 \leq j \neq k \leq n}\left\|\left(A_{k}+A_{j}\right) x\right\|^{2} \\
& \leq \sum_{k=1}^{n}\left\langle A_{k}^{*} A_{k} x, x\right\rangle+\sum_{1 \leq j \neq k \leq n}\left(\alpha\left(\frac{1}{\sqrt[\alpha]{4}}\right)^{r}+(1-\alpha)\left\|\left(A_{k}+A_{j}\right) x\right\|^{\frac{2 r}{1-\alpha}}\right)^{\frac{1}{r}} \\
& =\sum_{k=1}^{n}\left\langle A_{k}^{*} A_{k} x, x\right\rangle \\
& +\sum_{1 \leq j \neq k \leq n}\left(\alpha\left(\frac{1}{\sqrt[\alpha]{4}}\right)^{r}+(1-\alpha)\left\langle\left(A_{k}^{*}+A_{j}^{*}\right)\left(A_{k}+A_{j}\right) x, x\right\rangle^{\frac{r}{1-\alpha}}\right)^{\frac{1}{r}} \\
& \leq \sum_{k=1}^{n}\left\langle A_{k}^{*} A_{k} x, x\right\rangle \\
& +\sum_{1 \leq j \neq k \leq n}\left(\alpha\left(\frac{1}{\sqrt[\alpha]{4}}\right)^{r}+(1-\alpha)\left\langle\left(\left(A_{k}^{*}+A_{j}^{*}\right)\left(A_{k}+A_{j}\right)\right)^{\frac{r}{1-\alpha}} x, x\right\rangle\right)^{\frac{1}{r}}
\end{aligned}
$$




$$
\begin{aligned}
& =\left\langle\sum_{k=1}^{n} A_{k}^{*} A_{k} x, x\right\rangle \\
& +\sum_{1 \leq j \neq k \leq n}\left(\left\langle\left(\alpha\left(\frac{1}{\sqrt[\alpha]{4}}\right)^{r} I+(1-\alpha)\left(\left(A_{k}^{*}+A_{j}^{*}\right)\left(A_{k}+A_{j}\right)\right)^{\frac{r}{1-\alpha}}\right) x, x\right\rangle\right)^{\frac{1}{r}} .
\end{aligned}
$$

The proof is finished by taking the superemum over all $x \in H$ with $\|x\|=1$.

Similarly to what we have done in the proof of Corollary 4, Theorem 5 implies the following.

Corollary 6. Let $A \in B(H)$. Then

$$
\|A\|^{2} \leq \frac{1}{2}\left\|A^{*} A+A A^{*}\right\|+2\left\|\left(\frac{1}{2}\right)^{4 r+1} I+\frac{1}{2}\left(A^{*} A\right)^{2 r}\right\|^{\frac{1}{r}} .
$$

Following the same arguments used in the proof of Theorem 5, we achieve the following lemma.

Lemma 7. Let $A_{1}, \ldots, A_{n} \in B(H)$. Then for any $0 \leq \alpha<1$,

$$
\begin{aligned}
\left\|\sum A_{k}\right\|^{2} \leq\left\|\sum_{k=1}^{n} A_{k}^{*} A_{k}\right\| & +\alpha n(n-1) \\
& +(1-\alpha)\left\|\sum_{1 \leq j \neq k \leq n}\left(\left(\frac{A_{k}+A_{j}}{2}\right)^{*}\left(\frac{A_{k}+A_{j}}{2}\right)\right)^{\frac{1}{1-\alpha}}\right\| .
\end{aligned}
$$

Proof. Let $x \in H$ be a unit vector. Lemma 2 and the approaches used in the proof of Theorem 5 give that

$$
\begin{aligned}
\left\|\sum_{k=1}^{n} A_{k} x\right\|^{2} & \leq \sum_{k=1}^{n}\left\langle A_{k}^{*} A_{k} x, x\right\rangle+\sum_{1 \leq j \neq k \leq n}\left\|\left(\frac{A_{k}+A_{j}}{2}\right) x\right\|^{2} \\
& \leq \sum_{k=1}^{n}\left\langle A_{k}^{*} A_{k} x, x\right\rangle \\
& +(1-\alpha) \sum_{1 \leq j \neq k \leq n}\left\langle\left(\frac{A_{k}+A_{j}}{2}\right)^{*}\left(\frac{A_{k}+A_{j}}{2}\right) x, x\right\rangle^{\frac{1}{1-\alpha}}+\alpha n(n-1) \\
& \leq\left\langle\sum_{k=1}^{n} A_{k}^{*} A_{k} x, x\right\rangle+\alpha n(n-1)
\end{aligned}
$$




$$
+(1-\alpha)\left\langle\sum_{1 \leq j \neq k \leq n}\left(\left(\frac{A_{k}+A_{j}}{2}\right)^{*}\left(\frac{A_{k}+A_{j}}{2}\right)\right)^{\frac{1}{1-\alpha}} x, x\right\rangle .
$$

Take the supremum over all unit vectors $x \in H$, we obtain our lemma.

Applying the above lemma with $\alpha=0$, we get inequality (2).

Let us use Lemma 7 to generalize some known inequalities. In particular, we get the following.

Lemma 8. Let $A_{1}, A_{2}, \ldots, A_{n} \in B(H)$ and $0 \leq \alpha<1$. Then

$$
\begin{aligned}
\left\|\sum_{k=1}^{n} A_{k}\right\|^{2}+(1-\alpha) \sum_{k=1}^{n}\left\|A_{k}\right\|^{\frac{2}{1-\alpha}} & \\
& \leq\left\|\sum_{k=1}^{n} A_{k}^{*} A_{k}\right\|+\alpha n(n-1)+(1-\alpha) \sum_{j, k=1}^{n}\left\|\frac{A_{k}+A_{j}}{2}\right\|^{\frac{2}{1-\alpha}} .
\end{aligned}
$$

Proof. Let $x \in H$ be a unit vector. Use the same manner we used in proving Lemma 7, we obtain

$$
\left\|\sum_{k=1}^{n} A_{k} x\right\|^{2} \leq\left\langle\sum_{k=1}^{n} A_{k}^{*} A_{k} x, x\right\rangle+\alpha n(n-1)+(1-\alpha) \sum_{1 \leq j \neq k \leq n}\left\|\left(\frac{A_{k}+A_{j}}{2}\right) x\right\|^{\frac{2}{1-\alpha}} .
$$

Take the supremum over all unit vectors $x \in H$, we conclude

$$
\begin{aligned}
\left\|\sum_{k=1}^{n} A_{k}\right\|^{2} \leq\left\|\sum_{k=1}^{n} A_{k}^{*} A_{k}\right\| & +\alpha n(n-1) \\
& +(1-\alpha) \sum_{j, k=1}^{n}\left\|\frac{A_{k}+A_{j}}{2}\right\|^{\frac{2}{1-\alpha}}-\sum_{k=1}^{n}\left\|A_{k}\right\|^{\frac{2}{1-\alpha}}
\end{aligned}
$$

Use Lemma 8, with $\alpha=0$, we get [Theorem 2.3, [1]]. Also, use the same lemma with $\alpha=0$ and $n=2$, we deduce [Inequality (5), [7]].

We end our results with the following.

Theorem 9. Let $A_{1}, \ldots A_{n} \in B(H)$. Then

$$
\left\|\sum_{k=1}^{n} A_{k}\right\|^{2} \leq\left\|\sum_{k=1}^{n} A_{k}^{*} A_{k}\right\|+\frac{1}{2}\left\|I+\sum_{1 \leq j \neq k \leq n} A_{j}^{*} A_{k}\right\|^{2}-\frac{1}{2} .
$$


Proof. For a unit vector $x \in H$, we have that

$$
\begin{aligned}
\left\|\sum_{k=1}^{n} A_{k} x\right\|^{2}= & \sum_{k=1}^{n}\left\langle A_{k}^{*} A_{k} x, x\right\rangle+\frac{1}{2}\left\|I x+\sum_{1 \leq j \neq k \leq n} A_{j}^{*} A_{k} x\right\|^{2}-\frac{\|x\|^{2}}{2} \\
& -\frac{1}{2}\left\|\sum_{1 \leq j \neq k \leq n} A_{j}^{*} A_{k}\right\| \\
\leq & \sum_{k=1}^{n}\left\langle A_{k}^{*} A_{k} x, x\right\rangle+\frac{1}{2}\left\|\left(I+\sum_{1 \leq j \neq k \leq n} A_{j}^{*} A_{k}\right)^{x}\right\|^{2}-\frac{1}{2} .
\end{aligned}
$$

By taking the supremum over all unit vectors $x \in H$, we complete the proof.

Using the cartesian decomposition of the operator $A \in B(H)$, Theorem 10 with $n=2$ implies

$$
\|A\|^{2} \leq \frac{1}{2}\left\|A^{*} A+A A^{*}\right\|+\frac{1}{2}\left\|I+\frac{A^{*} A-A A^{*}}{2}\right\|^{2}-\frac{1}{2} .
$$

\section{References}

[1] S. Dragomir, Norm and numerical radius inequalities for sums of bounded linear operators in Hilbert spaces, Ser. Math. Inform, 22, No. 1 (2007), 61-75.

[2] S. Dragomir, Norm inequalities for sequences of operators related to the Schwarz inequality, J. Inequal. Pure and Appl. Math., 7, No. 3 Art. 97 (2006).

[3] S. Dragomir, Some Schwarz type inequalities for sequences of operators in Hilbert spaces, Bull. Austral. Math. Soc., 73, (2006), 17-26, doi: 10.1017/S0004972700038582.

[4] G. Hardy, J. Littlewood, and G. Pólya, Inequalities, Second Edition, Cambrridge University Press, Cambridge (1952).

[5] F. Kittaneh, Norm inequalities for sums and differences of positive operators, Lin. Alg. and Appl., $\mathbf{3 8 3}$ (2004), 85-91, doi: 10.1016/j.laa.2003.11.023. 
[6] F. Kittaneh, Notes on some inequalities for Hilbert space operators, Pub1. Res. Inst. Math. Sci., 24 (1988), 283-293, doi: 10.2977/prims/1195175202.

[7] F. Kittaneh, Numerical radius inequalities for Hilbert space operators, Studia Math., 168, No. 1 (2005), 73-80, doi: 10.4064/sm168-1-5. 